\title{
COVID-19 outbreak among physicians at a Canadian curling bonspiel: a descriptive observational study
}

\author{
Kelly W. Burak MD MSc(Epid), Sampson Law MSc, Chris Rice MDes, Jia Hu MD MSc, \\ Christopher I. Fung MD, Allan K.H. Woo MD, Kevin Fonseca PhD, Amanda L.S. Lang PhD, \\ Jamil N. Kanji MD, Bonnie L. Meatherall MD MSc
}

Abstract

Background: Transmission of severe acute respiratory syndrome coronavirus 2 (SARS-CoV-2) is known to occur among individuals who congregate in large groups, especially during indoor activities. Our objective was to provide a detailed clinical description of an outbreak of coronavirus disease 2019 (COVID-19) that occurred after a sporting and social event during the early days of the pandemic.

Methods: We conducted a descriptive study of a curling bonspiel in Edmonton held on Mar. 11-14, 2020. We used standardized interviews between Apr. 17 and May 5, 2020, to collect demographic data, travel history, symptoms (type, onset and duration), selfreported testing results for SARS-CoV-2 ribonucleic acid (RNA) using reverse transcription polymerase chain reaction (RT-PCR), and clinical outcomes. We also obtained results of convalescent SARS-CoV-2 immunoglobulin G serology.

Results: All 73 curlers (55 active health care workers) who participated in the bonspiel were interviewed for the study. Convalescent SARS-CoV-2 immunoglobulin G serology was completed in 62 (85\%) participants. Of the 73 participants (55 [75\%] male, median age 51 [range 26-79] yr, 58 [79\%] physicians), 40 curlers (55\%) tested positive for SARS-CoV-2 RNA by RT-PCR; an additional 16 participants developed symptoms but had negative swabs or were not tested ( 14 were probable cases), for a $74 \%$ attack rate (confirmed or probable cases). Anosmia with ageusia or dysgeusia occurred in 39 of 54 (72\%) confirmed or probable cases. The clinical course was mild in most participants (1 emergency visit, no hospital admissions). Transmission likely occurred from multiple individuals with minor nonspecific symptoms during the event, possibly during shared meals.

Interpretation: The $74 \%$ attack rate (confirmed or probable cases) highlights the infectivity of SARS-CoV-2 during sporting and social events. This reinforces the need for public health measures (masking, physical distancing and limiting the size of social gatherings) during future waves of COVID-19 in Canada.

anada confirmed its first case of the novel coronavirus disease 2019 (COVID-19), a respiratory illness caused by severe acute respiratory syndrome coronavirus 2 (SARS-CoV-2), on Jan. 25, 2020. ${ }^{1}$ As of Dec. 7, 2020, there were more than 420000 confirmed cases and nearly 13000 deaths in Canada. ${ }^{1}$ The World Health Organization (WHO) declared a global pandemic on Mar. 11, $2020,{ }^{2}$ which was also the date that physicians from around Canada gathered for a curling bonspiel in Edmonton (63rd Annual Western Canadian Medical Bonspiel, Mar. 11-14, 2020). Curling is a winter sport that originated in Scotland, but today $90 \%$ of all curlers worldwide come from Canada. ${ }^{3}$ At the onset of the event, there were only 24 confirmed cases of COVID-19 in Alberta (Figure 1). These cases were directly associated with international travel, and no known community spread had been documented.

In early March 2020, local public health guidelines in Alberta included limiting indoor gatherings to groups of less than 250 people, ${ }^{4}$ and there were no recommendations for using face masks in public places. During the bonspiel, enhanced safety measures included use of hand sanitizers, discouraging pre- and postgame handshakes, and sanitizing curling stones with disinfectant wipes between games. Despite these measures, an outbreak of COVID-19 occurred after the event. The pandemic has greatly affected organized sport around the world, ${ }^{5}$ and the experience at this bonspiel aided Curling Canada in its development of a return-to-play guideline. $^{6}$

Competing interests: None declared.

This article has been peer reviewed.

Correspondence to: Kelly Burak, kwburak@ucalgary.ca

CMAJ Open 2021. DOI:10.9778/cmajo.20200115 


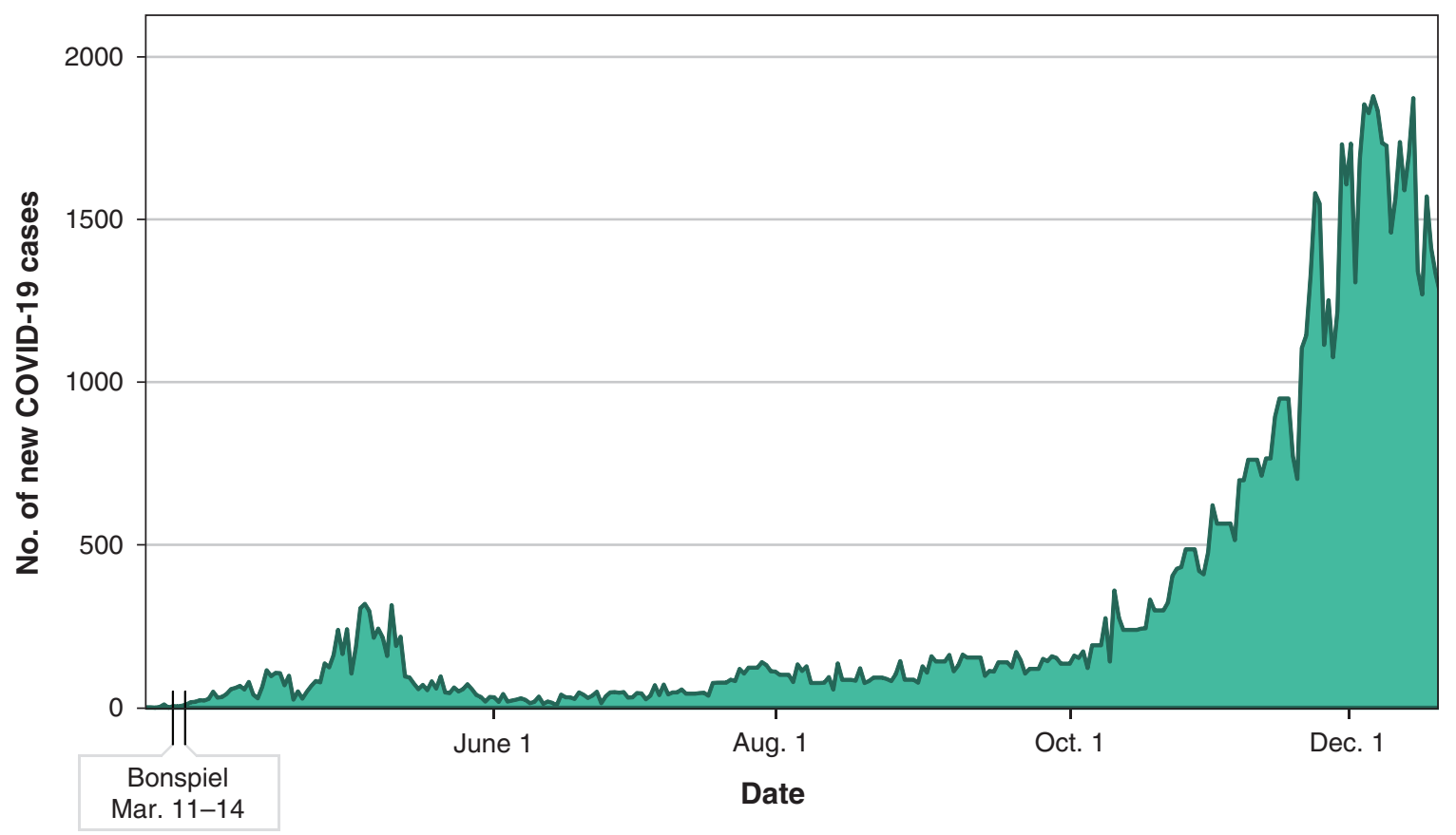

Figure 1: Confirmed cases of coronavirus disease 2019 (COVID-19) in Alberta, Canada, from Mar. 5 to Dec. 20, 2020. Created using publicly available data on COVID TRACKER (www.chi-csm.ca; accessed 2020 Dec. 22).

The objective of this study was to provide a detailed description of the onset, frequency and duration of symptoms experienced by the bonspiel participants, as well as the selfreported results of reverse transcription polymerase chain reaction (RT-PCR) testing immediately after the event and convalescent serologic testing for SARS-CoV-2, to highlight the infectivity of the virus during social and sporting events.

\section{Methods}

\section{Study design, setting and participants}

From Calgary, we conducted a descriptive observational study involving bonspiel participants between Apr. 17 and May 5, 2020. All curlers who attended a bonspiel on Mar. 11-14, 2020, were eligible for the study. The bonspiel was open to health care professionals from across Canada, although participating curlers were mainly physicians from Alberta, Saskatchewan, Manitoba and Ontario.

\section{Data sources}

A questionnaire was developed by K.W.B., B.L.M., S.L. and C.R., which focused on the main risk factor (international travel) and common symptoms reported in the literature ${ }^{7,8}$ and lay press ${ }^{9}$ at the time (Appendix 1, available at www.cmaj open.ca/content/9/1/E87/suppl/DC1). The questionnaire was piloted with 4 participants, and was refined and used by authors K.W.B. and B.L.M. to conduct telephone interviews (Apr. 17 to May 5, 2020). Data collected included demo- graphic characteristics, occupation, residence, travel history in the 28 days before the bonspiel, participation in social activities at the bonspiel, the onset and duration of symptoms, clinical outcomes and self-reported SARS-CoV-2 testing results. We chose to record 28 days of travel before the bonspiel based on what was known at the time about the incubation period of the virus and the emergence of symptoms (i.e., that symptoms can rarely develop more than 14 days after exposure) ${ }^{10}$ In addition, we recorded duration of self-isolation, meetings or patient encounters conducted before entering isolation, and awareness of transmission to family members, coworkers or patients.

Public health authorities, in the Canadian provinces where participants resided, were responsible for contact tracing, collecting upper respiratory tract samples and testing for SARSCoV-2 ribonucleic acid (RNA) by RT-PCR according to local protocols. Testing for SARS-CoV-2 immunoglobulin G (IgG) was conducted at Alberta Precision Laboratories using an initial screen with the Abbott SARS-CoV-2 IgG immunoassay (Abbott Laboratories) and confirmed using the Liaison SARS-CoV-2 S1/S2 IgG assay (DiaSorin).

\section{Case definitions}

Symptomatic participants were defined as "confirmed cases" if they had laboratory-confirmed results for SARS-CoV-2 using RT-PCR of upper respiratory tract samples (nasal, nasopharyngeal or throat), at any point during the course of their symptoms. Participants with symptoms who tested negative, 
or who were not tested, were categorized as a "probable cases" if they met the Public Health Agency of Canada (PHAC) interim national case definition criteria or the $\mathrm{WHO}$ criteria (Appendix 1). ${ }^{11,12}$

\section{Statistical analysis}

Survey responses were captured by K.W.B. and B.L.M. using Qualtrics XM survey software (Qualtrics). Before analysis, the survey information was double-checked for accuracy by K.W.B., B.L.M. and S.L. We summarized the data descriptively, with continuous variables presented as median and range or interquartile range (IQR), and categorical variables are summarized as counts and percentages. We used the Pearson $\chi^{2}$ test to compare symptomatic and asymptomatic groups. Analysis was performed using SPSS version 26 (IBM).

\section{Ethics approval}

The Conjoint Health Research Ethics Board at the University of Calgary approved this study (Ethics ID REB20-0514). Participants received a copy of the consent form by email and provided verbal consent by telephone. Serology was performed as part of a validation study (University of Alberta REB no. Pro00099818).

\section{Results}

All 73 curlers were eligible for the study and all consented to telephone interviews. Of the 73 curlers, 58 were physicians (6 retired). The median age was 51 (range 26-79) years, with one-quarter being above the age of $60 ; 55$ (75\%) were male. Eighteen teams (each with 3 to 5 curlers) competed during the bonspiel.

Fifty-six participants developed symptoms (Table 1), with 40 (55\%) of the 73 participants testing positive for SARS-CoV-2 by RT-PCR during their illness, including 6 individuals whose first RT-PCR was negative or indeterminate. Of the 16 symptomatic participants for whom RT-PCR was negative or not done, 14 met the PHAC or WHO definition for a probable case (Appendix 1), and 2 had nonspecific symptoms that did not meet criteria for a suspected or probable case by either definition (Figure 2).

\section{Symptoms}

The most common symptoms described among the 54 confirmed and probable COVID-19 cases were nasal congestion or rhinorrhea in 44 (81\%), cough in $41(76 \%)$, anosmia with or without dysgeusia in $39(72 \%)$, myalgia in 38 (70\%), headache in $33(61 \%)$ and fatigue or
Table 1: Demographic characteristics, travel history, and curling and social activities of bonspiel participants who did and did not develop symptoms

\begin{tabular}{|c|c|c|c|c|}
\hline \multirow[b]{2}{*}{ Variable } & \multicolumn{3}{|c|}{ No. (\%) of participants* } & \multirow[b]{2}{*}{$\begin{array}{c}p \\
\text { value }\end{array}$} \\
\hline & $\begin{array}{c}\text { Total } \\
n=73\end{array}$ & $\begin{array}{c}\text { Asymptomatic } \\
n=17\end{array}$ & $\begin{array}{c}\text { Symptomatic } \\
n=56\end{array}$ & \\
\hline \multicolumn{5}{|c|}{ Demographic characteristics } \\
\hline Age, yr, median (IQR) & $51(39-60)$ & $54(49-68)$ & $51(39-60)$ & \\
\hline \multicolumn{5}{|l|}{ Age distribution, yr } \\
\hline $20-29$ & $4(5)$ & $1(6)$ & $3(5)$ & \\
\hline $30-39$ & $18(25)$ & $2(12)$ & $16(29)$ & \\
\hline $40-49$ & $9(12)$ & $2(12)$ & $7(12.5)$ & \\
\hline $50-59$ & $23(32)$ & $5(29)$ & $18(32)$ & \\
\hline $60-69$ & $14(19)$ & $4(24)$ & $10(18)$ & \\
\hline $70-79$ & $5(7)$ & $3(18)$ & $2(4)$ & \\
\hline Sex, female & $18(25)$ & $3(18)$ & $15(27)$ & \\
\hline \multicolumn{5}{|l|}{ Occupation } \\
\hline Physician† & $58(79)$ & $13(76)$ & $45(80)$ & \\
\hline $\begin{array}{l}\text { Other health care } \\
\text { worker }\end{array}$ & $3(4)$ & $0(0)$ & $3(5)$ & \\
\hline Other & $12(16)$ & $4(24)$ & $8(14)$ & \\
\hline \multicolumn{5}{|l|}{ Province of residence } \\
\hline Alberta & $46(63)$ & $10(59)$ & $36(64)$ & \\
\hline Saskatchewan & $20(27)$ & $6(35)$ & $14(25)$ & \\
\hline Ontario & $4(5)$ & $1(6)$ & $3(5)$ & \\
\hline Manitoba & $3(4)$ & $0(0)$ & $3(5)$ & \\
\hline \multicolumn{5}{|c|}{ International travel within 4 weeks } \\
\hline Total & $27(37)$ & $8(47)$ & $19(34)$ & 0.7 \\
\hline United States & $20(27)$ & $7(41)$ & $13(23)$ & \\
\hline $\begin{array}{l}\text { Caribbean/Central } \\
\text { America }\end{array}$ & $3(4)$ & $1(6)$ & $2(4)$ & \\
\hline Africa & $1(1)$ & $0(0)$ & $1(2)$ & \\
\hline Asia & $1(1)$ & $0(0)$ & $1(2)$ & \\
\hline Europe & $2(3)$ & $0(0)$ & $2(4)$ & \\
\hline \multicolumn{5}{|c|}{ Activities during bonspiel } \\
\hline \multicolumn{5}{|l|}{ Curling } \\
\hline Thursday & $71(97)$ & $16(94)$ & $55(98)$ & 0.4 \\
\hline Friday & $68(93)$ & $16(94)$ & $52(93)$ & 0.9 \\
\hline Saturday & $63(86)$ & $12(71)$ & $51(91)$ & 0.4 \\
\hline \multicolumn{5}{|l|}{ Social events } \\
\hline $\begin{array}{l}\text { Wednesday } \\
\text { reception }\end{array}$ & $57(78)$ & $12(71)$ & $45(80)$ & 0.4 \\
\hline Thursday bowling & $34(47)$ & $7(41)$ & $27(48)$ & 0.6 \\
\hline Saturday banquet & $46(65)$ & $9(53)$ & $37(66)$ & 0.3 \\
\hline Hotel breakfasts & $48(66)$ & $10(59)$ & $38(68)$ & 0.5 \\
\hline $\begin{array}{l}\text { Buffet lunches at } \\
\text { curling rink }\end{array}$ & $67(92)$ & $12(71)$ & $55(98)$ & 0.002 \\
\hline $\begin{array}{l}\text { Note: IQR = interquartile ra } \\
\text { *Unless stated otherwise. } \\
\text { †Includes } 6 \text { retired physicia }\end{array}$ & & & & \\
\hline
\end{tabular}




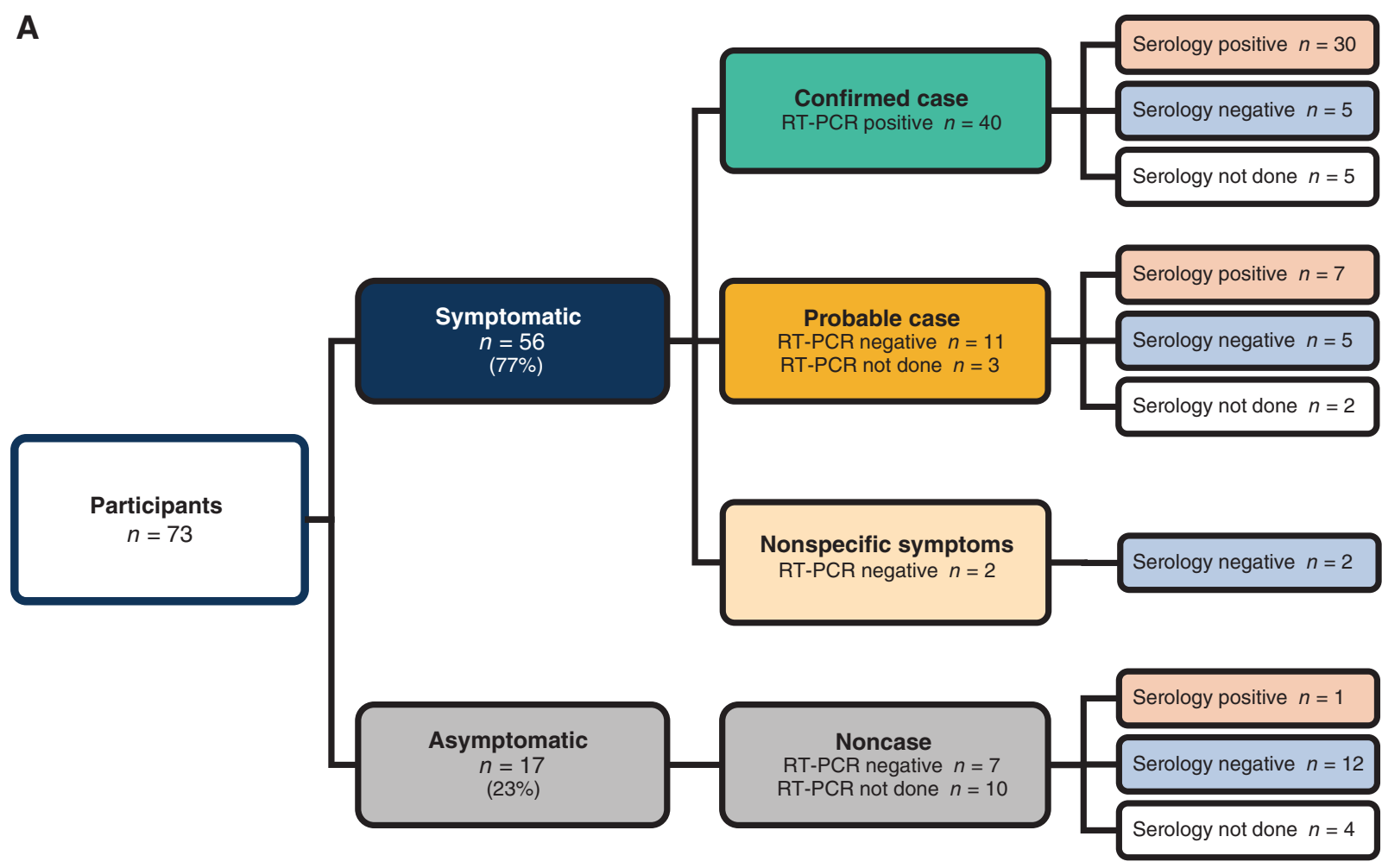

B

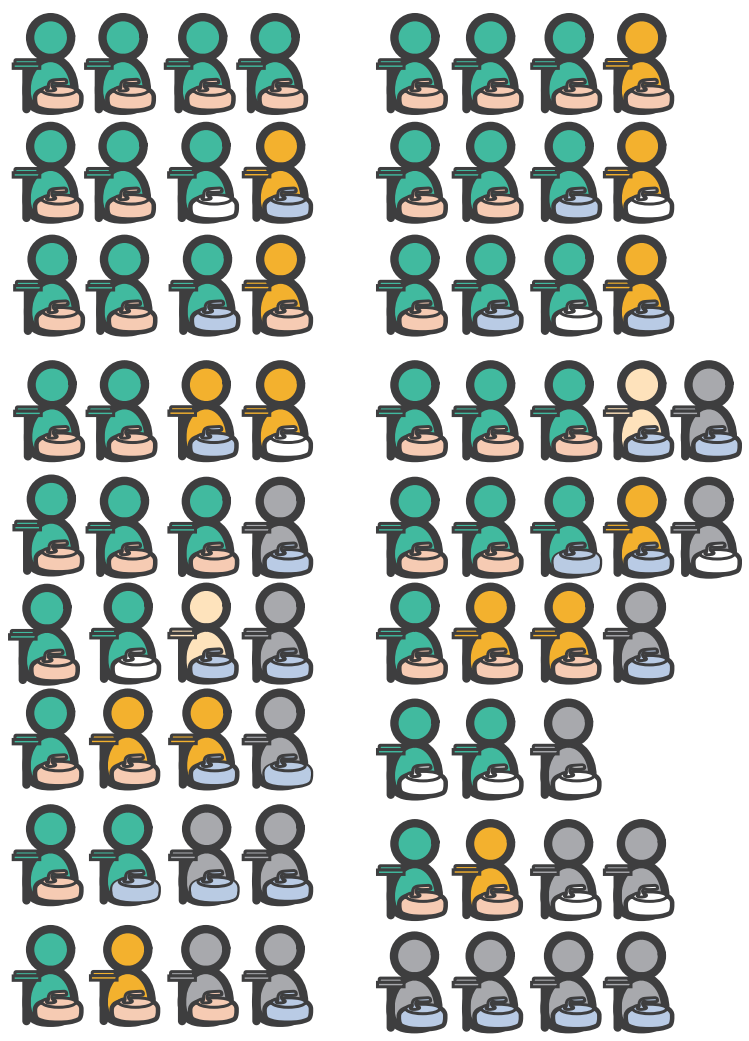

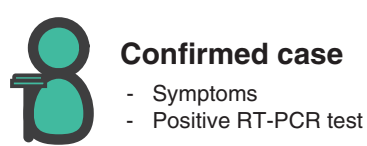

Probable case

- Symptoms

- Negative or no RT-PCR test

\section{Nonspecific}

- Nonspecific symptoms - Negative RT-PCR test

\section{Noncase}

- No symptoms

- Negative or no RT-PCR test

\section{Serology positive}

\section{Serology negative}

Serology not done

Figure 2: (A) Number of bonspiel participants with symptoms (confirmed cases, probable cases and nonspecific symptoms) or asymptomatic (noncases) and their serology results. (B) Number of bonspiel participants with symptoms (confirmed cases, probable cases and nonspecific symptoms) or asymptomatic (noncases) and their serology results, grouped by teams of 3 to 5 players. Note: RT-PCR $=$ reverse transcription polymerase chain reaction. 
exhaustion in 33 (61\%) (Figure 3A). Some symptoms (night sweats, productive cough, dyspnea and gastrointestinal symptoms) tended to occur later in the course (Figure 3B). Thirtytwo participants reported anosmia, which was associated with ageusia (loss of taste) or dysgeusia (altered taste) in 24 (75\%) participants. In 1 confirmed positive case, anosmia was the only symptom. Although anosmia could be present at onset of illness, the median onset was at 4 (IQR 2.0-5.3) days. At the time of data collection, all but 4 participants had fully recovered their sense of smell.

The clinical course was mild for most. Only 3 individuals formally sought advice from a physician. One participant was assessed in the emergency department, where chest radiography was normal and blood work showed only mild lymphopenia. No participants were admitted to hospital, and there were no deaths. Three days after conclusion of the event, we became aware of the first confirmed case, leading to the isolation of all curlers within 72 hours (Figure 4). The median isolation for curlers was 16 (range 10-30) days.

\section{Transmission during and after event}

From the beginning of the event, the median time to symptom onset was 6 (IQR 4.5-8) days. In retrospect, 10 participants identified that they had mild symptoms during the bonspiel (Figure 4). None of these individuals had symptoms consistent with the clinical case definition used by public health authorities at the time, and 3 had travelled internationally before the event. These individuals reported mild, nonspecific symptoms, including fatigue, myalgia, sore throat, nasal congestion, rhinorrhea, diarrhea and indigestion, and 2 reported mild cough. Two curlers withdrew from the event after the first day and 1 did not participate on the final day because of their mild symptoms.

Four curlers developed symptoms more than 14 days after the onset of the event (Figure 4). Three of these curlers were spouses of other confirmed cases, indicating possible secondary transmission in the home. Although formal contact tracing was not performed, participants reported that 35 family members developed symptoms consistent with COVID-19 (12 reportedly tested positive). Only 3 of these family members were present at the bonspiel, indicating that most of them likely acquired COVID-19 in the home. Forty bonspiel participants reported having meetings or seeing patients before entering isolation, and 6 reported being aware of a coworker or patient subsequently testing positive for COVID-19.

\section{Risk factors for transmission}

Eleven participants reported international travel in the 2 weeks before the bonspiel (14\% in symptomatic v. $18 \%$ in asymptomatic participants, $p=0.7$ ), with travel to the United States being the most common destination. An analysis of curling and social events at the bonspiel (Table 1) highlighted that a significantly greater proportion of symptomatic participants attended the buffet lunches at the curling rink $(98 \% \mathrm{v}$. $71 \%, p=0.002)$. Of the 18 teams participating, only 1 team had no cases (Figure 2B), and no members of this team attended the buffet lunches or other social events.

\section{Serology}

A total of 62 curlers (85\%) completed SARS-CoV-2 serology at a median of 121 (range 31-222) days after the event. Of the 40 confirmed cases, 30 also had positive serology, but serology was negative in 5 participants with positive RT-PCR testing, including 1 participant whose only symptom was anosmia and who did not develop IgG antibodies 67 days after the event (Figure 2). Of the 14 probable cases, 7 had positive serology and in 5 participants serology was negative. The 2 participants with nonspecific symptoms had negative serology. In the 17 asymptomatic individuals, serology was negative in 12 participants and was positive in 1 individual (collected $123 \mathrm{~d}$ after the end of the bonspiel). The 1 team without cases all tested negative for antibodies to SARS-CoV-2.

\section{Interpretation}

Seventeen of 18 teams who participated in the bonspiel had at least 1 confirmed case of COVID-19, with 1 team having all 4 members being confirmed cases and 6 teams having all members as confirmed or probable cases (Figure $2 \mathrm{~B}$ ). The high attack rate we observed ( $74 \%$ of curlers were confirmed or probable cases), even with some enhanced safety measures in place, highlights the infectivity of SARS-CoV-2 during sporting and social events. As our standardized interviews took place within 5-8 weeks of the participants' illness, and our group contained a large number of health care workers, we were able to collect an accurate and detailed description of the spectrum of clinical disease caused by SARS-CoV-2 in this cohort. Based on our analysis, the clinical spectrum of COVID-19 varied widely from person to person and included symptoms of influenza-like illness, but also atypical symptoms such anosmia, noted in almost three-quarters of our confirmed or probable cases.

Our serology data had some interesting findings. In the 1 confirmed case with only anosmia, substantial IgG antibodies had not developed 2 months after the event. Other studies have linked the severity of symptoms to the probability of antibodies developing. ${ }^{13}$ In the other 4 confirmed cases with negative serology, serology was done quite late $(115,194,207,220 \mathrm{~d})$, and the results may represent the waning of antibodies titres over time. ${ }^{14}$ The 1 asymptomatic case with positive serology never had RT-PCR testing, and this may represent another case from the bonspiel, as asymptomatic infection can occur. ${ }^{15}$ However, we cannot exclude the possibility that this health care worker was exposed to SARS-CoV-2 through another occupational or community exposure after the bonspiel.

Our data suggest there may have been more than 1 introduction event into this cohort, rather than a single index case (Figure 4); however, this is difficult to confirm without phylogenetic analysis of the positive cases. This does highlight the importance of recommendations for individuals with any symptoms, even if mild, to stay away from work and social activities. The feasibility of studying the transmission dynamics of this outbreak was made challenging by the social nature of the bonspiel. However, our data suggest that attending buffet lunches in the curling rink lounge may have been important 
A

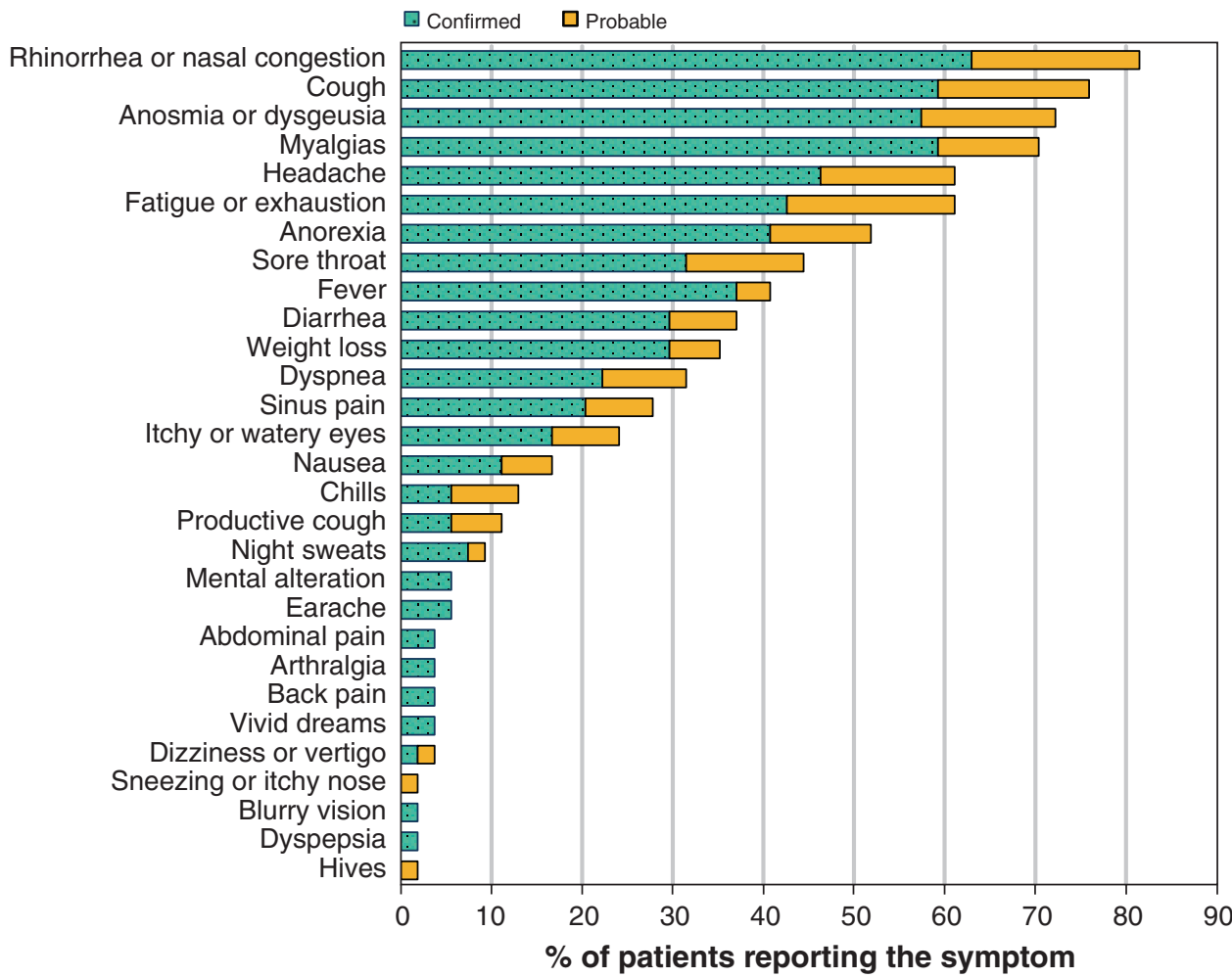

B

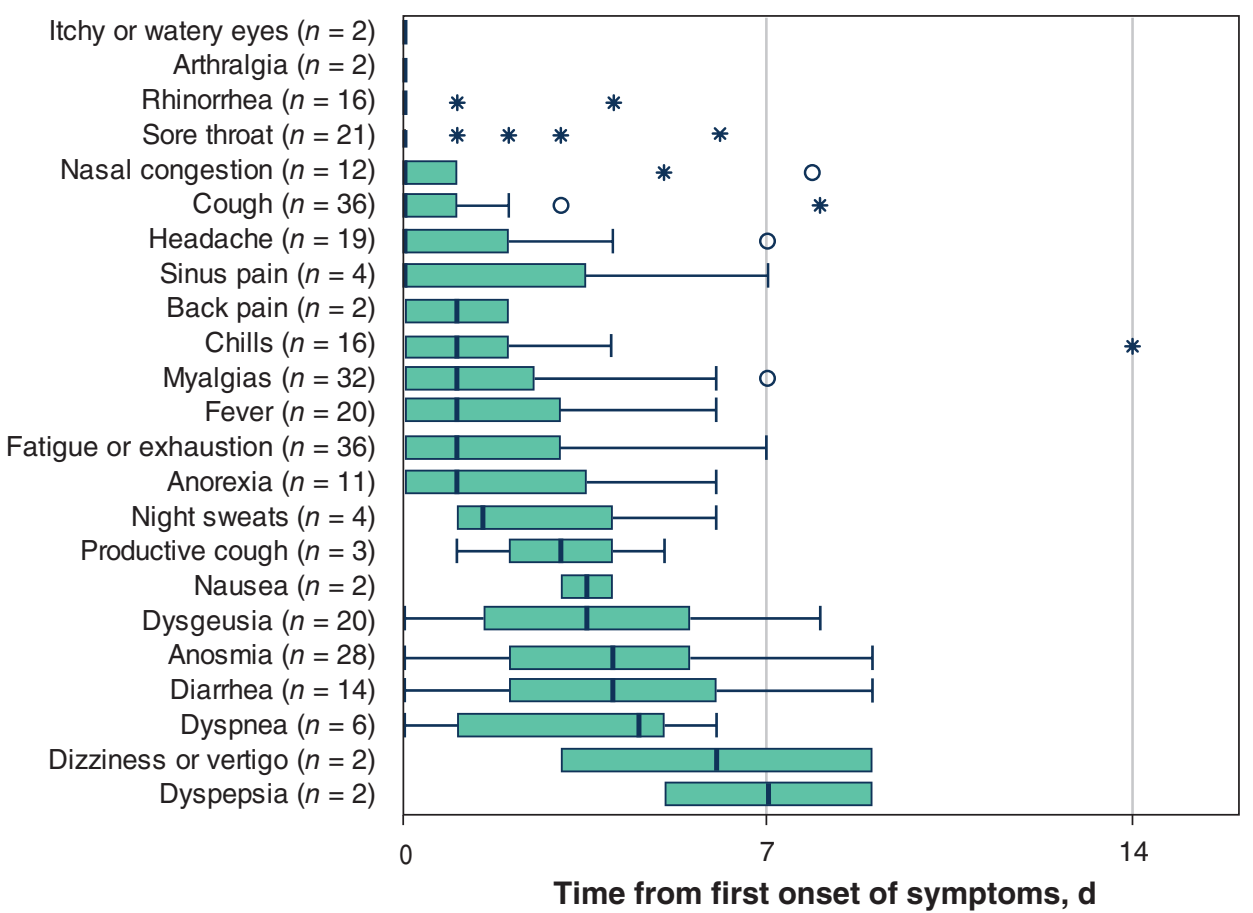

Figure 3: (A) Frequency of symptoms among confirmed ( $n=40)$ and probable cases $(n=14)$ of coronavirus disease 2019 (COVID-19). (B) The median onset of common symptoms among confirmed and probable cases. Note: Data presented in Figure 3B include only those participants who recalled the precise onset and resolution of their symptoms. Dysgeusia (altered taste) includes 18 with ageusia (loss of taste). Box and whiskers plots show the medians and interquartile ranges (IQRs). Outliers within $3 \times$ IQR (open circles) or outside $\left(^{*}\right)$. 


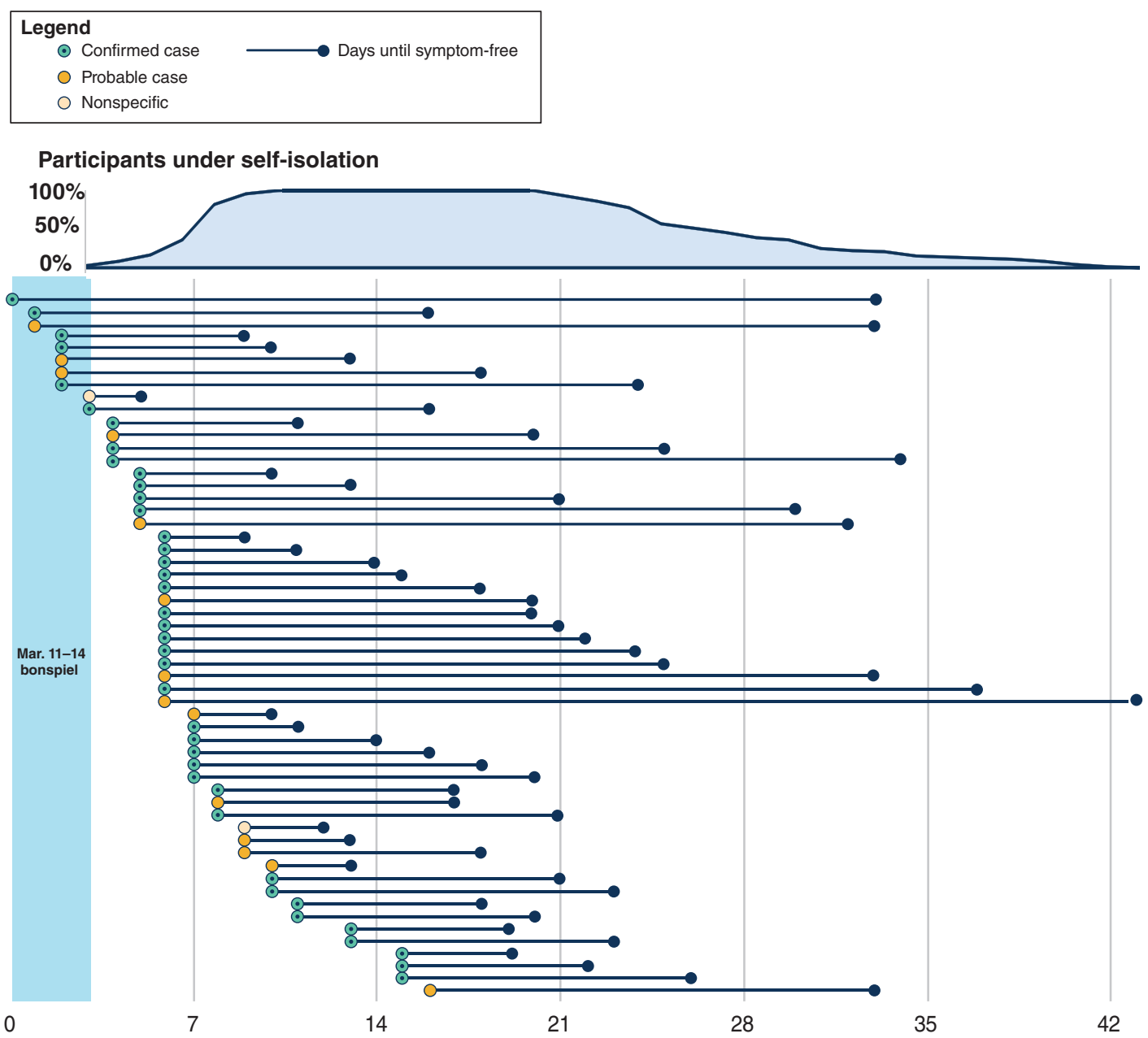

Figure 4: In relation to the event, the onset and duration of symptoms in confirmed cases $(n=40)$, probable cases $(n=14)$ and those with nonspecific symptoms $(n=2)$, along with the percentage of participants under self-isolation in the days from the end of the bonspiel.

for transmission. Interestingly, the single team with no confirmed cases did not attend any social events outside of their curling games, suggesting that social activities associated with sporting events may be as, or more, important for transmission. Analysis of transmission clusters, or "superspreader events," has shown that they usually occur indoors, often when people are speaking loudly or are in proximity to each other, usually without the use of masks. ${ }^{16}$ These are important considerations as we move toward reinitiating organized sports, especially when they are combined with socializing before or after the activity.

Our study also highlights that community transmission can be an important mode of infection in health care workers. As of Dec. 8, 2020, 4483 Albertans who self-identify as health care workers have tested positive for COVID-19, but it is not known how many infections were through occupational or community exposure (www.alberta.ca/stats/covid-19-alberta -statistics.htm). Health care workers worry about the risk of transmitting SARS-CoV-2 to their family members, ${ }^{17}$ and in our study, symptomatic curlers reported that 35 family members developed symptoms of COVID-19. Although we did not perform formal contact tracing, only 3 of these symptomatic family members attended the bonspiel as a guest, suggesting a high rate of secondary transmission in the home during self-isolation. A preprint meta-analysis of 40 studies found an overall attack rate in the household of $18.8 \%$ (95\% confidence interval [CI] $15.4 \%-22.2 \%) .{ }^{18}$

During the early part of the pandemic, fever and cough were recognized as the predominant symptoms of COVID19. ${ }^{7}$ However, by the time of our event there were emerging reports of atypical symptoms involving the gastrointestinal tract $^{8}$ and the loss of sense of smell. ${ }^{9}$ A recent systematic review and meta-analysis of 10 studies $(n=1627)$ showed olfactory dysfunction in $52.7 \%$ (95\% CI 29.6\%-75.2\%) of patients with COVID-19. ${ }^{19}$ Another study involving 803 health care workers from the Netherlands with mild 
symptoms (90 positive for SARS-CoV-2) found that anosmia was the most important symptom in a predictive model to diagnose SARS-CoV-2. ${ }^{20}$ Our study shows that anosmia tended to occur a few days after onset of other symptoms (Figure 3B) and can occur without other symptoms such as nasal congestion or rhinorrhea. However, waiting to isolate until this specific symptom is present could lead to risk of spread to others. Comparing clinical definitions of suspected and probable cases (Appendix 1), the PHAC uses fewer symptoms in its interim definition, ${ }^{12}$ and the WHO elevates suspected cases to probable based on the presence of anosmia or ageusia alone. ${ }^{11}$ Our experience highlights that many symptoms of COVID-19 are nonspecific and that establishing a diagnosis based on clinical symptoms can be difficult, especially during cold and flu season. We believe that anosmia can be helpful in distinguishing COVID-19 from other viral infections, and we encourage the PHAC to follow the WHO's lead to include anosmia in its clinical definition.

Future phylogenetic analysis of our outbreak may provide further clues regarding transmission dynamics. Several curlers are participating in a study to follow their SARS-CoV-2 antibody titres over time. A follow-up survey is planned to examine long-term symptoms in our bonspiel participants.

\section{Limitations}

Limitations of our study include our small sample, which may limit generalizability. RT-PCR testing was not available for all symptomatic participants, as access to RT-PCR testing was variable by province in the early days of the COVID-19 pandemic in Canada. Similar to RT-PCR testing, serologic testing was not available for all participants. We needed to wait for validated serologic tests to become available, and the timing of serologic testing was not standardized between participants. Ideally, we would want paired serologic samples, with the acute sample 4 weeks after exposure, and a repeat convalescent sample 6 weeks later showing an increasing titre. Not having early RT-PCR and timely paired serology for all participants left us to rely on clinical case definitions, which may underestimate (missed asymptomatic cases) or overestimate (symptoms attributed to COVID-19 due to other viral illnesses) our true attack rate. We recognize the retrospective nature of our data collection and that emerging reports of atypical symptoms may have influenced reporting of symptoms. Furthermore, we did not use a validated test of anosmia. However, during the outbreak when participants were contacted (by C.I.F., A.K.H.W., K.W.B. and J.H.) about the need for isolation and testing, they were encouraged to journal their symptoms carefully. We acknowledge that some of the self-reported variables, viewed as being sensitive, may have led to respondent bias. Complete data on public health contact tracing of each participant were not available to our study team, and, therefore, we do not know the true secondary spread of SARS-CoV-2 from the bonspiel participants.

\section{Conclusion}

This study describes the variable clinical presentation of COVID-19 among a group of health care providers in a com- munity setting. In addition to fever and cough, unusual symptoms such as anosmia are common and should be included in clinical case definitions of COVID-19. Most importantly, our study highlights the infectivity of SARS-CoV-2, how efficiently the virus spreads between asymptomatic or paucisymptomatic individuals gathering for social or sporting events, and the need to follow public health recommendations as we consider continuing social and sporting activities during or after future waves of COVID-19.

\section{References}

1. Coronavirus disease 2019 (COVID-19): epidemiology update. Ottawa: Public Health Agency of Canada; updated 2020 Dec. 21. Available: https://health -infobase.canada.ca/covid-19/epidemiological-summary-covid-19-cases.html (accessed 2020 Oct. 22).

2. Timeline: WHO's COVID-19 response. Geneva: World Health Organization. Available: www.who.int/emergencies/diseases/novel-coronavirus-2019/ interactive-timeline\# (accessed 2020 Dec. 8).

3. The Curling Canada brand [main page]. Available: http://brand.curling.ca (accessed 2020 Oct. 22).

4. Chief medical officer of health COVID-19 update - March 12, 2020. Edmonton: Government of Alberta; 2020 Mar. 18. Available: www.alberta.ca/release. cfm?xID=69840592E937F-0ADF-8CA5-AF6EDFF1E53B5AC3 (accessed 2020 Dec. 8).

5. Dove J, Gage A, Kriz P, et al. COVID-19 and review of current recommendations for return to athletic play. RI Med 7 (2013) 2020;103:15-20.

6. Return-to-play guidelines: a resource for boards of directors, volunteers and managers of Canadian curling rinks. Orleans (ON): Curling Canada; 2020. Available: www.curling.ca/files/2020/07/CC-Manual_Return-to-Play_CAN_EN.pdf (accessed 2020 Oct. 22)

7. Chen N, Zhou M, Dong X, et al. Epidemiological and clinical characteristics of 99 cases of 2019 novel coronavirus pneumonia in Wuhan, China: a descriptive study. Lancet 2020;395:507-13.

8. Jin X, Lian J-S, Hu J-H, et al. Epidemiological, clinical and virological characteristics of 74 cases of coronavirus-infected disease 2019 (COVID-19) with gastrointestinal symptoms. Gut 2020;69:1002-9.

9. Rabin RC. Lost sense of smell may be peculiar clue to coronavirus infection. The New York Times 2020 Mar. 22, updated 2020 Mar. 26. Available: www.ny times.com/2020/03/22/health/coronavirus-symptoms-smell-taste.html (accessed 2020 Mar. 30).

10. Lauer SA, Grantz KH, Bi Q, et al. The incubation period of coronavirus disease 2019 (COVID-19) from publicly reported confirmed cases: estimation and application. Ann Intern Med 2020;172:577-82.

11. WHO COVID-19 Case definition. Geneva: World Health Organization (WHO); 2020. Available: www.who.int/publications/i/item/WHO-2019 -nCoV-Surveillance_Case_Definition-2020.2 (accessed 2020 Oct. 22).

12. Interim national case definition: coronavirus disease (COVID-19). Ottawa: Public Health Agency of Canada; updated 2020 Apr. 2, 2020. Available: www.canada.ca/ en/public-health/services/diseases/2019-novel-coronavirus-infection/health -professionals/national-case-definition.html (accessed 2020 Oct. 22).

13. Long Q-X, Tan X-J, Shi Q-L, et al. Clinical and immunological assessment of asymptomatic SARS-CoV2 infections. Nat Med 2020;26:1200-4.

14. Murchu EO, Byrne P, Walsh KA, et al. Immune response following infection with SARS-CoV-2 and other coronaviruses: a rapid review. Rev Med Virol 2020 Sept. 23 [Epub ahead of print]. doi: 10.1002/rmv.2162.

15. Kronbichler A, Kresse D, Yoon S, et al. Asymptomatic patients as a source of COVID-19 infections: a systematic review and meta-analysis. Int 7 Infect Dis 2020;98:180-6.

16. Meyerowitz EA, Richterman A, Gandhi RT, et al. Transmission of SARSCoV-2: a review of viral, host, and environmental factors. Ann Intern Med 2020 Sept. 17 [Epub ahead of print]. doi: 10.7326/M20-5008.

17. Souadka A, Essangri H, Benkabbou A, et al. COVID-19 and healthcare worker's families: behind the scenes of frontline response. EClinicalMedicine 2020;23:100373.

18. Madewell ZJ, Yang Y, Longini IM Jr, et al. Household transmission of SARS-CoV-2: a systematic review and meta-analysis of secondary attack rate [preprint]. medRxiv 2020 Aug. 1. doi: 10.1101/2020.07.29.20164590.

19. Tong JY, Wong A, Zhu D, et al. The prevalence of olfactory and gustatory dysfunction in COVID-19 patients: a systematic review and meta-analysis. Otolaryngol Head Neck Surg 2020;163:3-11.

20. Tostmann A, Bradley J, Bousema T, et al. Strong associations and moderate predictive value of early symptoms for SARS-CoV-2 test positivity among healthcare workers, the Netherlands, March 2020. Euro Surveill 2020;25: 2000508 .

Affiliations: Division of Gastroenterology and Hepatology (Burak), Department of Medicine, and Physician Learning Program (Burak, Law, 
Rice), Continuing Medical Education and Professional Development, and Cumming School of Medicine, University of Calgary; Alberta Health Services $(\mathrm{Hu})$; Department of Community Health Sciences (Hu), Department of Medicine, Cumming School of Medicine, University of Calgary, Calgary, Alta.; Department of Radiology and Diagnostic Imaging (Fung), Faculty of Medicine and Dentistry, University of Alberta, Edmonton, Alta.; Division of Orthopedic Surgery (Woo), Department of Surgery, College of Medicine, University of Saskatchewan, Saskatoon, Sask.; Provincial Laboratory for Public Health (Fonseca), Alberta Precision Laboratories; Department of Microbiology, Immunology and Infectious Diseases (Fonseca), Cumming School of Medicine, University of Calgary, Calgary, Alta.; Roy Romano Provincial Lab (Lang), Saskatchewan Health Authority, Regina, Sask.; Division of Infectious Diseases (Kanji), Department of Medicine, Faculty of Medicine and Dentistry, University of Alberta; Provincial Laboratory for Public Health (Kanji), Alberta Precision Laboratories, Edmonton, Alta.; Division of Infectious Diseases (Meatherall), Department of Medicine, Cumming School of Medicine, University of Calgary, Calgary, Alta.

Contributors: Kelly Burak, Bonnie Meatherall, Sampson Law, Chris Rice, Christopher Fung, Allan Woo and Jia Hu contributed to the study design. Kelly Burak and Bonnie Meatherall contributed to data collection (interviews), and Kevin Fonseca, Jamil Kanji and Amanda Lang contributed to data collection (serology). Sampson Law, Kelly Burak and Bonnie Meatherall contributed to data analysis. Chris Rice and Sampson Law contributed to data visualization. Kelly Burak, Bonnie Meatherall and Sampson Law contributed to writing the first draft and revisions. All authors approved the final version to be published and agreed to be accountable for all aspects of the work.

Funding: This project received in-kind support from the Physician Learning Program.

Content licence: This is an Open Access article distributed in accordance with the terms of the Creative Commons Attribution (CC BY-NC-ND 4.0) licence, which permits use, distribution and reproduction in any medium, provided that the original publication is properly cited, the use is noncommercial (i.e., research or educational use), and no modifications or adaptations are made. See: https://creativecommons.org/licenses/by-nc-nd/4.0/

Data sharing: All data will not be made publicly available. Researchers who require access to the study data can contact the corresponding author for further information (kwburak@ucalgary.ca).

Acknowledgement: The authors acknowledge Dr. Tyler Williamson from the Centre for Health Informatics at the Cumming School of Medicine for assistance in creating Figure 1.

Supplemental information: For reviewer comments and the original submission of this manuscript, please see www.cmajopen.ca/content/9/1/ E87/suppl/DC1. 